\title{
110 \\ From Experience Feedback Towards an Evolutive Memory for Participatory Design
}

\author{
Markus D. Durstewitz \\ Man-Machine Systems Laboratory, IMAT-MMS, University of Kassel \\ Ph.D. Research Project carried out with Eurisco, Toulouse \\ Eurisco, 4 ave Edouard Belin, F-31400 Toulouse \\ FRANCE \\ Markus.Durstewitz@onecert.fr
}

\begin{abstract}
Previous experience plays a key-role in design problem-solving. Therefore we formalized experience feedback and access in order to improve the design process and the quality of products so designed. Groups of designers use shared design documents in their work. Therefore we introduced the concept of experience folders as a document classification scheme. It enables real-time experience capture and the externalization of design rationale so it can be shared with others. Cross-referencing experiences with contact information about people involved in design facilitates communication between the actual design team and the company's experts. So the experience folder is used to maintain competencies and to update the corporate design knowledge in an evolutive design memory.
\end{abstract}

KEY WORDS experience feedback, design rationale, corporate memory, competence management

\section{BACKGROUND}

Today, we experience the creation of the information society. Information is empowering people and organizations. A company in the information age needs a tremendous amount of data, information, and knowledge to be competitive on the world market. Intellectual resources as experiences and know-how become a decisive factor in business (Conklin 1996). But a company cannot store everything and if so, it would be nearly impossible to access problem relevant information afterwards. Thus, we have to make a choice in storing data, information, and knowledge ${ }^{1}$. Particularly in the iterative design of complex systems such as nuclear power plants or aircraft the need for an effective assistance in information management becomes obvious.

The aim of this research project is to improve product quality and performance and the underlying design

\footnotetext{
1 we know about the differences of data, information, and knowledge according to different meaning and interpretation levels of a message (see Aamodt, 1995). But in this paper we use information as a generic term for all meaning and interpretation levels.
} 
process itself by better satisfaction of the designers' experience demands through the formalization and exploitation of experience feedback in design. This enables the capitalization of know-how, and the creation of a living design memory (Terveen, 1993) as part of an electronic corporate memory (ECM). It is utilized to represent and access design knowledge and design rationale (Gruber 1990; Steels 1993). This must not be a complex system. Already simple means can make the difference. Mark Ackerman showed in a field study with Answer Garden how experts by "just" answering questions contribute to the augmentation of the organizational memory within the habitual work frame (Ackerman 1994).

\section{A MEMORY FOR DESIGN}

Corporate memory is a generic concept to save, represent, and share essential knowledge and by this way to support cooperation in a multiple task and multiple user environment. The concept includes organizational, functional, and social aspects of the work, the worker and the workplace (CoMe 1994).

Today, we have the paradox that on one hand the designers claim information and on the other hand they cannot master the information overflow. Therefore ECM should rather inform the designer on his/her demand (Kidd 1994) and assist him/her in information access and retrieval (Boy 1995) than paralyze his/her decision creativity by presenting overwhelming amounts of information on the computer. ECM should not only be an information storage but a means for coordination and communication in problem-solving (Bannon 1996). The representation and measurement of the relevance of information is significant for the success of an ECM. Therefore consequent user feedback is needed (Boy 1991) and continuous update of the memory so that it can evolve with change in design.

We see two approaches of interaction for creating an ECM:

(1) no limits approach or «just do it ».

People are allowed to write down whatever they want and to exchange information with everybody without any restriction. Then we suppose the knowledge collection will be maximized. The problem is now to find a way how this knowledge collection can be organized and classified in order to be reusable in future problem-solving.
(2) top-down approach or « do just this ».

People have to follow rigid regulations. They have to write specific documents according to specific tasks and these documents then are approved in order to be distributed in a restricted way. We suppose that this approach which is very common in nowadays companies will generate a predefined classification structure but minimize the information flow.

In real world applications, designers of ECM must find a compromise between both approaches. In our research project we followed a collaborative system design approach where the designers and their interactions are in the center of interest.

\subsection{Document management as problem- solving in design}

We studied the design modification process, its procedures and the formal exchange of knowledge via documents. Therefore we observed and followed a group of designers in their everyday work. Our observation was accompanied by interviews. In particular, we focused on information needs and the use of design documents with respect to a specific design problem. Thus we paid attention on both, how the designers perceive the design process and influences of norms and regulations, and how they act in the real problemsolving process. This led us to the creation of a user and problem-oriented information flow model. We identified three main tasks in the designer's problemsolving process: (1) identification and observation of a problem, (2) analysis and interpretation of the problem, and (3) proposal and application of a valuable solution. Each task is related to specific types of documents like (1) incident report, technical follow up, (2) test result notification, technical specification, and (3) service bulletin. We recognized that the biggest part of design information is represented by these documents (see also Karsenty 1995). New documents arrive every day and one of the designer's biggest problems is how to dispose of all the documents on his/her desk without losing important information relevant to the actual problem. Nevertheless design rationale, the reasons why a solution was chosen, and the expert's know-how, the lessons learned from experience, mostly are not documented. In this situation it becomes very difficult to share knowledge and to anticipate problems. The designer has no time to think about and to decide whether or not an information might be of interest for a 
colleague in another department or for a future project. Here, document classification becomes the central issue. First we tried classification by chronological order according to the date of incoming documents. This was not sufficient with regard to information exchange and transfer. In fact, designers tend to classify documents according to specific types of problems. That is the reason why at least we elaborated the experience folder concept as a document classification scheme. The folder structure corresponds to the problem-solving process used by the designers.

\subsection{Formalization of experience feedback}

Our study'showed that it is common for designers to reuse already validated and approved solutions of former similar design problem cases - if they are available! In this way, analogical and case-based reasoning is one of the basic techniques of design and in particular of the design-modification process. The exploitation of experience requires formal means for capture and exchange of information related to the prior case if we do not want to limit experience to the individual memory of the designer him/herself but allow experience exchange across departments and, eventually, across the whole company.

Herein we considered special events, abnormal situations, dysfunctionning, or incidents that occur while using a product. Then designers do not only assist in trouble-shooting but they also have to reevaluate product quality, safety, and reliability. They have to elaborate a repair solution or eventually a design modification.

Incident reporting systems and formalized user feedback provide the information that is necessary to decide whether or not a design modification will be undertaken. The formalization of experience feedback by using experience folders provides the information that is necessary to find a valuable solution based on examples of prior cases. Furthermore the folder can be used to formulate the lessons learned from experience. Therefore the designers propose a design rule and/or recommendation which is validated by a committee of wise men before it is applied in the design process (see REAIMS project, Durstewitz 95). Keeping a link between rules and experience cases the original experience can assist the designer in problem-solving by illustrating valuable details about why and how to perform modifications while applying a rule and/or recommendation.

\subsection{Real time experience capture}

We continued our investigation with the question: how can the individual designer capture his/her design experience effectively (that means in respect of reuse), and how can he/she transfer this experience to the community of potentially concerned designers.

The problem of experience capture is related to the management of information and documents concerning design problems. We saw the need for a tool that enables the designer to structure and to classify documents according to the evolution of a specific design project, and which enables him/her to add important information, particularly design rationale and explanation to the considered document.

We introduced the experience folder as a means for simple (user oriented) and effective (use-oriented) project management and experience capture. An experience folder represents a structured and classified collection of documents ${ }^{2}$ concerning one specific design problem or "case ». First, we created paper forms and then we implemented electronic experience folders linked to the company's design database. Documents are classified in chapters, sections, and subsections according to the steps in the resolution of a typical design problem. This enables the designer to capture experience in real-time, when it arises - and facilitates the later access to problem-relevant information.

The experience folder assists the designer in keeping track of the project status. He/she can organize and classify documents and his/her personal annotations, summaries, etc. in a problem-oriented way. We based the structure of the experience folder on the model corresponding to the designers' document-exchange procedure in a typical design modification process.

\subsection{Active competence management}

Problems are addressed to people, and people solve problems. In this way it is very important to have a connection between problems and people. To know who knows what becomes crucial. Therefore we investigated about the creation of the company's white and yellow pages as a means for competence management.

We proposed a structured problem and task-oriented personal competence page. It represents a kind of electronic $\mathrm{CV}$ of each designer. Then we only have to

\footnotetext{
${ }^{2}$ electronically each folder has its equivalent and each document can be represented by a file: text, illustration, image, sound, or video etc.
} 
make link between problems and experts by adding a list of people involved in the design project to the experience folder. The list is attached to the different folder sections and refers to the personal competence page of each person involved in the specific task of the problem-solving process. Thus the folder can be used by the design team as a kind of project agenda and eases connections to competent experts.

\section{INTERACTION WITH THE ECM}

Up to now, we have implemented the folder structure models so that it can be coupled to a data-base. When a request for work or change arrives the designer consults the data base for similar problem cases. These can be used as examples in problem-solving. If no case was found or a former case cannot be applied without major changes then the designer creates a new experience folder. Therefore he/she fills in electronic forms (or paper forms the time the system is not completely deployed) by answering to standard questions like what has happened when and where, what is the probable cause of the problem, what do you intend to do to fix the problem and why, what are your recommendations. The system analyses the answer written in natural language style and proposes an indexation for the experience folder based on a predefined code lists. If a field keeps empty, the system will ask for more detail. At least the designer validates the proposed indexation or can change it using the code lists. If necessary the designer himself can extend the code list and add a new key word. The extra work of folder creation pays back in short term because it can be used as project protocol or agenda and avoids long search for documents or contacts.

The next steps are the validation and the deployment of an electronic experience folder system:

(1) computer supported update and upgrade of documentation.

(2) computer supported competence management introducing electronic white-and yellow-pages

(3) integration of electronic dictionaries and thesaurus, for example, definitions of profession specific terms

(4) automatic coherence check in documents, for example, in the use of abbreviations
(5) intranet deployment will offer the possibility of hyperlinks, for example, towards document references or expert contacts

\section{CONCLUSION}

Documents are the main information source and communication medium in design. Therefore designers need an effective assistance for document management. They reuse experience cases in design problem solving thus design rationale and the lessons learned from experience must be externalized and documented.

We elaborated by the systematic involvement of the designers a conceptual framework for experience feedback and competence management: the experience folder concept. It supports the acquisition, storage, and distribution of data, information, and knowledge. It is

(consequent integration of user feedback enables the measurement of the knowledge base quality and its improvement following the law of supply and demand.

(real time experience capture during the problemsolving process (in vivo) guarantees up-to-date information in the knowledge base.

〈process- and problem-oriented experience case collection assists the designer in analogical reasoning by illustrating example solutions.

〈direct links between the problem and the people involved in solving the design problem facilitates the contact with experts and enables the creation and management of virtual project networks.

The deployment of an information system is merely related to the company's organizational structure (Prinz, 1993) and social context. The efforts can only be estimated roughly because costs will depend on the technical state of the working environment as well as on social and human factors which have to be evaluated.

Information sharing will lead to unexpected and severe consequences in the companies' organization if we do not try to take into account seriously the close relation between technical, individual, and social aspects of work. For us it is a fact that any change on one of those 
elements will imply changes on the others (Israel, 1997).

Without a common effort and coordination of organizational changes, electronic document management initiatives, intelligent data base development, and intranet solutions we will not be able to create an ECM.

\section{REFERENCES}

Aamodt, A. and Nygaard, M. (1996) Different roles and mutual dependencies of data, information, and knowledge - an AI perspective on their integration, in Data and Knowledge Engineering, North-Holland

Ackerman, M. S. (1994) Augmenting the Organizational Memory: A Field Study of Answer Garden, in $C S C W^{\prime} 94$, New York, ACM Press

Bannon, L. and Kuutti, K. (1996) Shifting Perspectives on Organizational Memory: From Storage to Active

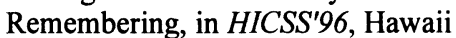

Boy, G. (1991) Computer Integrated Design, in Sociomedia (ed. E. Barrett), MIT Press, pp. 507-531

Boy, G. (1995) Design Rationale and Supportability: An Agent-Oriented Corporate Memory Approach, in COOP'95, Juan-Les-Pins, France

CoMe (1994) - in Newsletter of the Corporate Memory Initiative (Eurisco, IIG Universität Freiburg, IFIT at University of Trondheim, and TCD Dublin) ed. Durstewitz, M. D., Toulouse, France

Conklin, J. (1996) Designing Organizational Memory: Preserving Intellectual Assets in a Knowledge Economy, Memorandum, Corporate Memory Systems Inc., Austin, Texas

Durstewitz, M.D., Boy, G., Heckmann J.P. (1995) Experience-Based Aircraft Design, in $H M I-A I-A S$ ' 95 , Toulouse

Gruber, T.R. (1990) Design knowledge and design rationale: A framework for representing, capture, and use, KSL technical report, Stanford University

Israel, R., Durstewitz, M., and Hollender, M. (1997) Social Context in ECM, Eurisco Working paper, Toulouse, France

Karsenty, L., Attipoe, A., Durstewitz, M., and Lafrique, D. (1995) La communication via documents dans les projets de conception, in 01Design'95, Autrans, France
Kidd, A. (1994) The Marks are on the Knowledge Worker, in proceedings of $\mathrm{CHI}^{\prime} 94$, pp. 186-191, ACM Press, New York

Prinz, W. (1993) TOSCA - providing organizational information to $\mathrm{CSCW}$ applications, in $E C S C W^{\prime} 93$, Kluwer, pp. 139-154

Steels, L. (1993) Corporate Memory Management, AIMemo 93-03, Vrije Universiteit Brussel, Belgium

Terveen, L.G., Selfridge, P.G., and Long, M.D. (1993) From "Folklore" to "Living Design Memory", in INTERCHI'93, pp 15-22, Amsterdam 